\section{Retinoblastoma Plus Lipomatosis: An Autosomal Dominant Syndrome}

Stefania Tenna, Mauro Barone, Pierluigi Gigliofiorito, Paolo Persichetti

Department of Plastic, Reconstructive, and Aesthetic Surgery Unit, Campus Bio-Medico University of Rome, Rome, Italy

\section{Correspondence: Mauro Barone}

Department of Plastic, Reconstructive, and Aesthetic Surgery Unit, Campus Bio-Medico University of Rome, Via Alvaro del Portillo 200, Rome, Italy

Tel: +622541220, Fax: +0622541936

E-mail:maurosabbarone@gmail.com

\section{Pierluigi Gigliofiorito}

Department of Plastic, Reconstructive, and Aesthetic Surgery Unit, Campus Bio-Medico University of Rome, Via Alvaro del Portillo 200, Rome, Italy Tel: +622541220, Fax: +622541936

E-mail: p.gigliofiorito@gmail.com

No potential conflict of interest relevant to this article was reported.

Received: 21 Feb 2014 • Revised: 30 May 2014• Accepted: 9 Jun 2014 pISSN: 2234-6163 • elSSN: 2234-6171

http://dx.doi.org/10.5999/aps.2014.41.6.785 • Arch Plast Surg 2014;41:785-787

Copyright (C) 2014 The Korean Society of Plastic and Reconstructive Surgeons

This is an Open Access article distributed under the terms of the Creative Commons

Attribution Non-Commercial License (http://creativecommons.org/licenses/by-nc/3.0) which permits unrestricted non-commercial use, distribution, and reproduction in any medium, provided the original work is properly cited.

A 43-year-old man, Caucasian, was admitted to our unit with a lump extending from the nape of the neck to the dorsal region between the shoulders (Figs. 1, 2). This mass had first appeared about 10 years prior to presentation at our institution and had since been increasing gradually in size. The patient had a history of hereditary bilateral retinoblastoma treated at the age of 1 year by enucleation of the right eye and radiation therapy on the contralateral eye; at the age of 18 years, recurrence of disease in the left temporo-zygomatic region was disposed and treated with radiation therapy and chemotherapy. Since then until recently, there had been no signs of recurrence.

At physical examination, lower neck swelling was detected with poorly defined margins, soft-elastic consistency, little shifting, and no signs of inflammation or pain.

Magnetic resonance imaging (MRI) (Fig. 3) revealed a significant thickening of the subcutaneous fat from the nape of the neck to the dorsal region between the shoulders, with two notable masses: the cranial, which measured $2.4 \mathrm{~cm} \times 5 \mathrm{~cm} \times 8 \mathrm{~cm}$ and the distal, in the dorsal region, $6.5 \mathrm{~cm} \times 12 \mathrm{~cm} \times 8 \mathrm{~cm}$. These formations, both well-encapsulated, had homogeneous hyperintense signals in the T1- and T2- weighted sequences with a reduction of the nonhomogeneous internal signal sequences obtained with the suppression of the fat signal with modest and nuanced post-contrast enhancement.

A preliminary diagnosis of multiple lipomatosis was taken. The procedure was performed under general anesthesia. A double surgical access was adopted: 1) the first, in the nuchal region, with enucleation en bloc of the swelling, which was partly submuscular; and 2) the second, in the dorsal region, created by using a tumescent liposuction technique through two incisions of $3 \mathrm{~mm}$ each with a drawing of about 600 $\mathrm{mL}$ of blood serum-fat emulsion.

The residual fatty emulsion was injected in the subsidence of the left temporo-zygomatic region.

The postoperative course was uneventful with good healing of the surgical wounds.

A histological examination of the nuchal mass and the dorsal liposuction confirmed the tissue as benign lipoma and lipomatosis tissue. The follow-up at 6 months was negative for the recurrence of the disease (Figs. 4, 5)

In order to document the presence of retinoblastoma, multiple endocrine neoplasia (MEN) 1 and lipomatosis were evaluated with an preoperative genetic exam to confirm the diagnosis of this rare and complex syndrome; in fact, hereditary predisposition to lipomas is observed in familial multiple lipomatosis and benign cervical lipomatosis (and can also be associated with mutations in the MEN1 and phosphatase and TENsin homolog

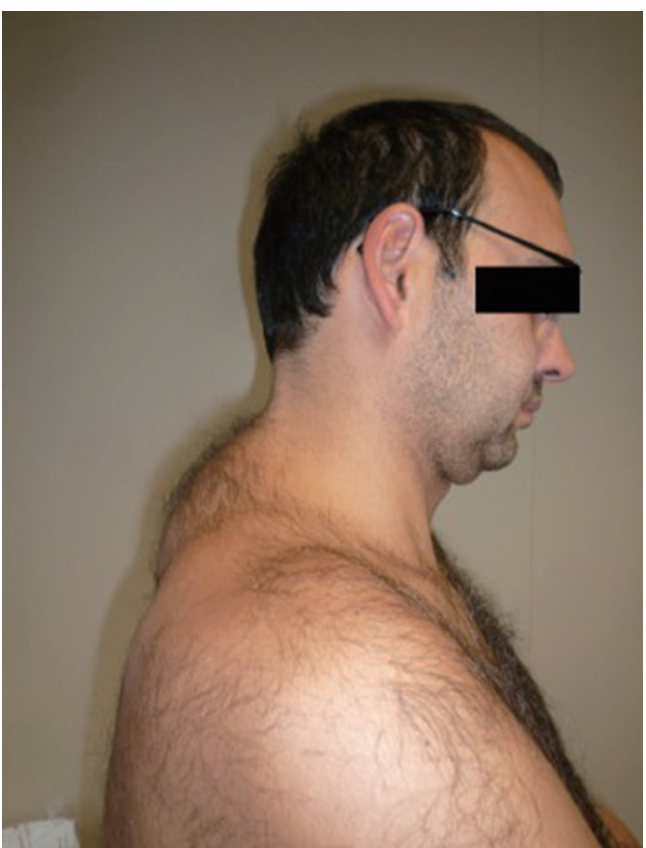

Fig. 1.

Preoperative situation. 
Fig. 2.

Preoperative situation.

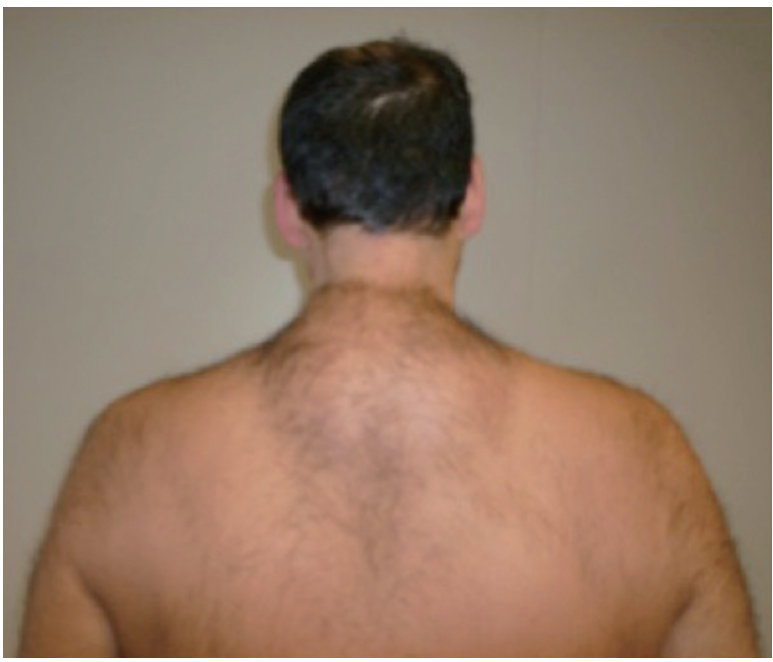

[PTEN] genes). In addition, a recent report indicates that a few patients with hereditary retinoblastoma also have lipomas. In the pedigree presented in this patient, the autosomal dominant transmission of multiple lipomas is linked to a mutant retinoblastoma (RB) 1 allele. Therefore, early diagnosis is very important because the progeny is surely affected.

Lipomas are the hamartomatous proliferation of mature fat cells; these are benign adipose tumors of the mesenchymal origin with a prevalence rate of 2.1:1,000 to 1:100; approximately $25 \%$ of the lipomas and their variants arise in the head and neck region.

Sometimes, lipomas are associated with syndromes such as multiple lipomatosis (lipoma over extremities and the trunk), Gardner's syndrome (intestinal polyposis, cysts, and osteomas), Dercum's disease (multiple painful subcutaneous lipomas), and Madelung's disease (lipomatosis of the neck, head, shoulders, and proximal extremities).

On the computed tomography (CT) scans, lipomas have the typical characteristics of homogeneous masses with few septations and a specific range of CT hounsfield unit (HU) values (usually between -50 $\mathrm{HU}$ and $-150 \mathrm{HU}$ ); further, they show no contrast enhancement. MRI can also accurately diagnose lipomas preoperatively by a comparison of signal intensity on the T1- and T2-weighted images.

Conventional lipomas have characteristic chromosomal abnormalities, often showing chromosomal rearrangements of $12 \mathrm{q} 14-15,6 \mathrm{p}$ and 13q9 [1]; in this regard, Genuardi et al. [2], reasserting the claims made by other authors, reported that lipomas occur frequently in patients with HBRB, a rare intraocular eye cancer, presenting in children before the age of 2 years, in which the germline

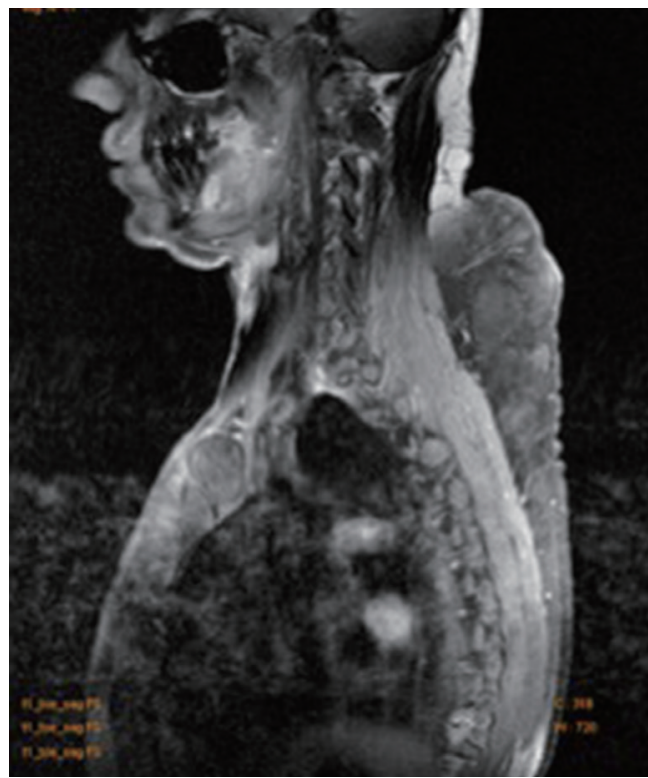

Fig. 3.

Magnetic resonance imaging.

mutations of the retinoblastoma gene ( $R B 1$ ) occur.

Mutations involve the deletions or translocation of the chromosome 13q14. The RB1 encodes the retinoblastoma protein, which is the central regulator of mammalian cell cycles, and in particular, it acts as a molecular switch of adipocyte differentiation.

In addition, multiple lipomas are observed in the context of hereditary neoplastic syndromes including those associated with mutations in the MEN1 and PTEN genes [1,2].

Treatment modalities for lipomas can be divided into nonexcisional and excisional therapy.

Surgical excision is still the main treatment of lipoma; Stebbins et al. [3] state that surgical excision is the treatment of choice for small lipomas. These authors further claim that in patients with large or multiple lipomas, excision scars may result in large and numerous scars; therefore, cosmetically more acceptable approaches, such as modified incisional approaches, liposuction, and intralesional laser therapy with or without liposuction, have been successful.

Pereira and Schonauer [4] have proposed to remove the lipoma through a small incision by using a pair of gynecological polyp forceps passed down a subcutaneous tunnel from an incision placed in an aesthetically advantageous site.

In addition to cosmetic surgery, liposuction is a therapeutic option in other conditions such as lipomas and lipomatosis. This procedure has been enhanced by several modifications; one of the most significant 


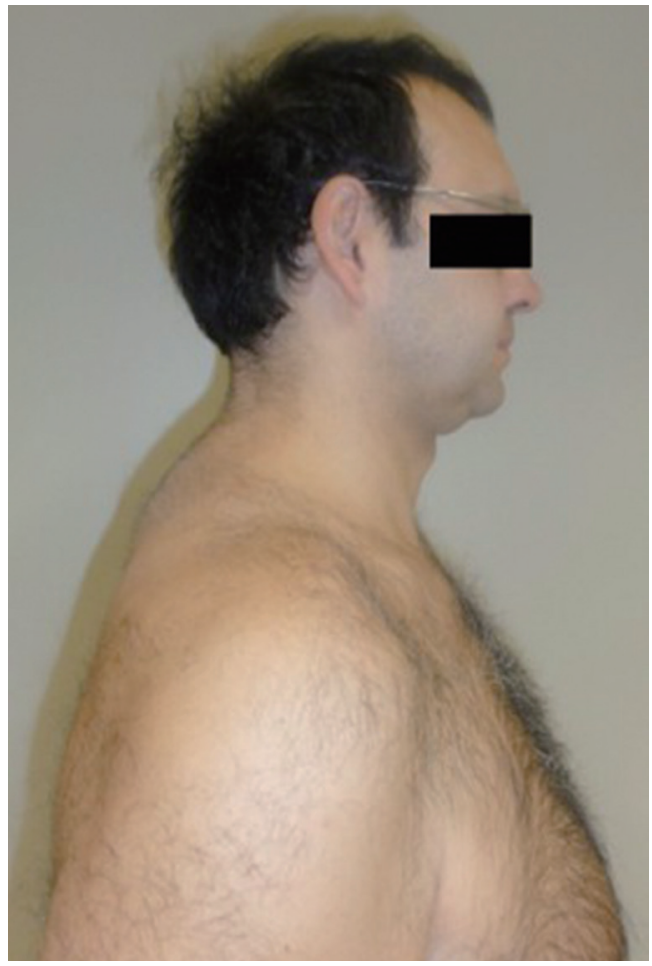

Fig. 4.

Cosmetic outcome

advances has been the use of the tumescent technique for removing fat. Also reporting the experience of previous works, Stebbins et al. [3] have stated that although liposuction has been used successfully to remove or reduce large lipomas, there are certain instances when complete excision is inhibited by lipomas with a more significant fibrous component. In this setting, these authors have asserted that laser lipolysis can be used to lyse fibrous connections of the lipoma, while liquefying the fat. It has been estimated that approximately $10 \%$ to $18 \%$ of the lipomas are incompletely removed using laser lipolysis with or without subsequent liposuction. However, given the minimal invasivity of the procedure, retreatment is usually well-tolerated.

We report the case of a patient with HBRB and multiple lipomas of the base of the neck and upper back. This association occurs in less than $4 \%$ of the subjects with HBRB. The treatment of lipomas in our patient consisted of a combined approach (surgical excision and tumescent liposuction) with excellent aesthetic results, respecting the principles of literature. An early diagnosis can help the patient to be aware of his disease, which could be transmitted to his children, despite there being no previous cases of the same disease in the family, as reported by the study of

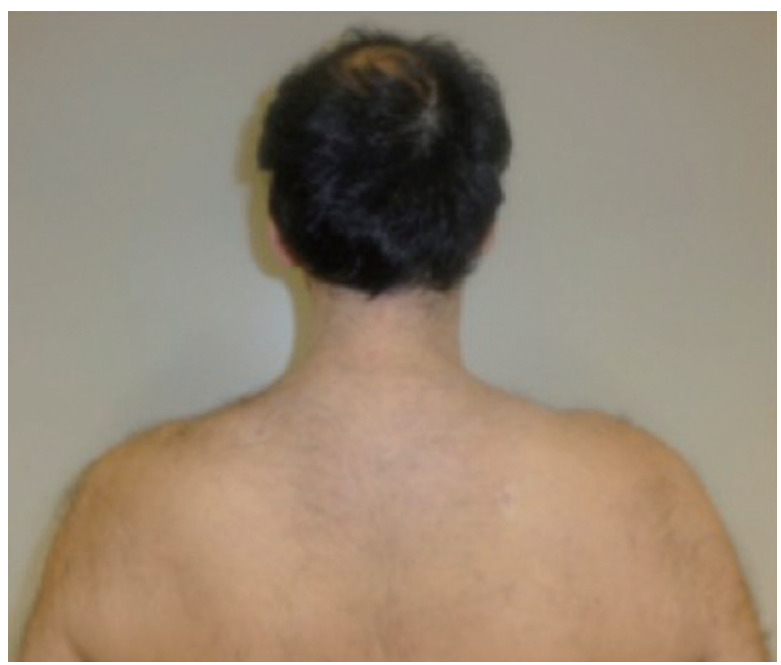

Fig. 5.

Cosmetic outcome.

Rieder et al. [5].

\section{References}

1. Li FP, Abramson DH, Tarone RE, et al. Hereditary retinoblastoma, lipoma, and second primary cancers. J Natl Cancer Inst 1997;89:83-4.

2. Genuardi M, Klutz M, Devriendt K, et al. Multiple lipomas linked to an RB1 gene mutation in a large pedigree with low penetrance retinoblastoma. Eur J Hum Genet 2001;9:690-4.

3. Stebbins WG, Hanke CW, Petersen J. Novel method of minimally invasive removal of large lipoma after laser lipolysis with $980 \mathrm{~nm}$ diode laser. Dermatol Ther 2011;24:125-30.

4. Pereira JA, Schonauer F. Lipoma extraction via small remote incisions. Br J Plast Surg 2001;54:25-7.

5. Rieder H, Lohmann D, Poensgen B, et al. Loss of heterozygosity of the retinoblastoma (RB1) gene in lipomas from a retinoblastoma patient. J Natl Cancer Inst 1998;90:324-6. 